\title{
Usage des traducteurs en ligne et enjeux affectifs de l'apprentissage de l'anglais en lycée
}

\author{
Aurélie Bourdais ${ }^{1, *}$ \\ ${ }^{1}$ ICAR (UMR 5191), Université Lumière Lyon 2, 69007 Lyon, France
}

\begin{abstract}
Résumé. Les émotions jouent un rôle non négligeable dans le processus d'apprentissage [1]. Le présent article a pour but d'explorer dans quelle mesure les usages des traducteurs en ligne par les lycéens sont liés aux enjeux affectifs relatifs à l'apprentissage de l'anglais en milieu institutionnel. Il s'appuie sur une étude dont l'objectif était d'observer le rôle des traducteurs en ligne dans le cadre d'activités de production écrite proposées à des lycéens en cours d'anglais. Des captures d'écran vidéo réalisées en salle multimédia ont donné à voir le processus de rédaction et plus particulièrement les recherches effectuées par les élèves. Les entretiens individuels effectués à la suite de cette séance de production écrite ont permis à 5 participants de visualiser la capture d'écran réalisée sur ordinateur et de la commenter. La méthodologie adoptée a permis une mise à distance des émotions ressenties lors de l'activité en vue d'en faciliter la verbalisation, mais le contexte interactionnel de l'entretien a également provoqué l'émergence de nouvelles émotions.
\end{abstract}

\begin{abstract}
Emotions play a significant role in the learning process [1]. The purpose of this article is to explore to what extent the uses of online translators by highschool students are linked to the emotional issues related to the learning of English in an institutional setting. It is based on a study, the goal of which was to observe the role of online translators within the context of writing activities proposed to highschool students during an English lesson. Video screenshots taken in a computer room have shown the writing process and more particularly the research carried out online by the students. The individual interviews carried out after the writing session enabled 5 participants to view and comment on the screenshot taken on the computer. The methodology adopted allowed a distancing of the emotions felt during the activity so as to verbalise them more easily, but the interactional context of the interview also triggered the emergence of new emotions.
\end{abstract}

\section{Introduction}

Les traducteurs en ligne, à la différence des dictionnaires en ligne ou des concordanciers de type Linguee, font aujourd'hui partie de la panoplie des lycéens. Nombre d'enseignants de

\footnotetext{
*a.bourdais@univ-lyon2.fr
} 
langues étrangères ont pu constater depuis une dizaine d'années que les élèves y avaient recours, souvent en dépit de leurs interdictions. En effet, une enquête par questionnaires menée auprès d'élèves de Première $(n=118)$ en 2017 a confirmé qu'une écrasante majorité d'entre eux utilise les traducteurs en ligne, quel que soit le niveau de compétences qu'ils estiment avoir atteint en anglais. Selon la même enquête, les élèves ont recours aux traducteurs en ligne non seulement pour comprendre ou pour écrire, mais plus encore à des fins de vérification. La vérification fait partie du processus rédactionnel tel qu'il a été décrit par Flower \& Hayes [2], au même titre que la génération d'idées ou la génération de texte, mais elle ne peut pas être réduite à sa dimension cognitive. En effet, les observations menées ont montré que les élèves vérifient souvent ce qu'ils savent déjà. Qu'elle renvoie à la crainte de faire une erreur ou à de la curiosité, la vérification peut également avoir une valeur affective très forte. La vérification relèverait alors autant de la sphère affective que de la sphère cognitive.

L'enjeu de la recherche présentée ici est d'observer certains des mécanismes émotionnels qui entrent en jeu lorsque les élèves ont recours aux traducteurs en ligne et de mieux comprendre les raisons pour lesquelles les lycéens ont fréquemment recours à ces ressources.

\section{Cadre théorique}

Les fondements de la recherche sur les émotions sont souvent associés aux travaux de William James [3], qui a décrit les émotions comme des mécanismes physiologiques déclenchés en réaction à un stimulus. La compréhension des émotions s'est complexifiée depuis la fin du dix-neuvième siècle et leur rôle dans l'apprentissage a fait l'objet de recherches spécifiques.

\subsection{Les émotions: quelques éléments de définition}

Selon Frijda [4] et Linnenbrink [5], une émotion peut être définie comme la réaction à un événement que l'individu considère comme important. Cette réaction le prépare à agir en cohérence avec son ressenti. Bien que les définitions du concept d'émotions soient multiples et parfois divergentes, la dimension subjective de cette expérience ainsi que la notion d'évaluation sont deux traits sur lesquels il semble y avoir un large consensus. Les émotions ne sont aujourd'hui généralement plus circonscrites à leurs manifestations physiologiques. Il est aujourd'hui reconnu que les émotions sont des mécanismes complexes constitués également de composantes psychique, comportementale et cognitive [6], remettant ainsi en question le dualisme cartésien [7]. En outre, les avancées récentes en neurosciences ont démontré que les émotions ne se limitent pas à la relation entre corps et cerveau, mais qu'elles sont indissociables de l'environnement [8]. Selon KerbratOrecchioni [9], les émotions doivent en effet être considérées comme des expériences intersubjectives, « intégrée(s) dans un processus relationnel »: l'émotion ressentie dépend de la personne avec laquelle on est engagé dans une interaction. Les émotions sont enfin des constructions sociales, dans le sens où leur interprétation dépend des interprétations qui ont pu être faites précédemment par d'autres individus [10].

La dimension motivationnelle [11] est parfois aussi évoquée. La motivation, selon qu'elle est intrinsèque et renvoie à la « réalisation d'une activité par le seul effet du plaisir ou de la satisfaction qu'elle génère » [12] ou bien extrinsèque ou instrumentale, c'est-à-dire liée aux bénéfices qui peuvent être tirés de l'activité ou des résultats de l'activité [13], jouerait ici un rôle non négligeable [5], en particulier dans un contexte d'apprentissage. 


\subsection{Emotions et éducation}

Le rôle des émotions dans l'apprentissage et l'enseignement est incontestable. Il ressort d'études menées en psychologie de l'éducation que les émotions peuvent être décrites selon leur objet, leur valence et leur activation [14].

Les émotions en lien avec l'éducation peuvent être classées selon l'objet à l'origine de l'émotion en question [14]:

- les émotions d'accomplissement sont liées à une activité d'accomplissement (plaisir ou ennui) ou au résultat d'accomplissement. Les émotions liées au résultat peuvent être prospectives (espoir, anxiété) ou rétrospectives (fierté, déception) ;

- les émotions épistémiques portent sur la connaissance (curiosité, confusion);

- les émotions sociales ont pour objet les relations avec l'enseignant ou les pairs ;

- les émotions thématiques sont liées au thème traité.

Il est également possible de classer les émotions selon:

- leur valence: les émotions sont positives lorsque le sujet éprouve du plaisir tandis que les émotions négatives sont causées par une expérience déplaisante ;

- leur activation: les émotions activantes nous incitent à agir, contrairement aux émotions désactivantes (cf. Fig. 1).

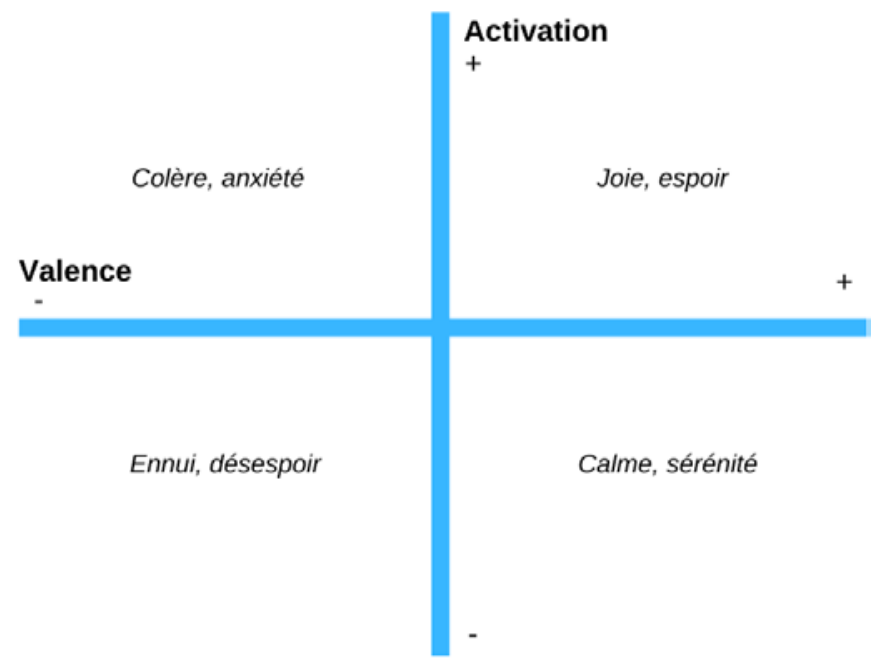

Fig. 1. Classification des émotions [15]

Ces classifications ont conduit à l'élaboration d'une théorie du contrôle et de la valeur des émotions d'accomplissement, selon laquelle les émotions dépendent d'une part de la perception du contrôle que l'apprenant a sur l'activité ou le résultat d'accomplissement, d'autre part de la valeur que l'apprenant accorde à cette activité ou à ce résultat [13-14]. Ces émotions influencent à leur tour le processus d'apprentissage. Les émotions d'accomplissement, comme toutes les émotions, sont donc liées à la subjectivité de l'apprenant ainsi qu'à son évaluation de la situation, ce qui confirme les relations étroites existant entre cognition et affect.

L'étude présentée ici s'attachera à répondre aux questions suivantes:

- Dans quelle mesure l'usage des traducteurs en ligne est-il lié aux émotions des lycéens, et plus spécifiquement aux enjeux affectifs de l'apprentissage de l'anglais? 
- Dans quelle mesure l'observation de traces du processus rédactionnel et le contexte interactionnel offrent-ils des indices sur les états émotionnels des lycéens?

\section{Présentation du protocole de recherche}

Les données ont été recueillies en deux étapes, dans le cadre d'une étude exploratoire menée dans un lycée de l'agglomération lyonnaise, auprès de lycéens de Première Technologique inscrits en section européenne. Ces élèves bénéficient d'un volume horaire d'enseignement d'anglais plus important que les élèves suivant un enseignement classique: à l'heure supplémentaire hebdomadaire d'enseignement de langue étrangère s'ajoute une heure de Discipline Non Linguistique, ou DNL (ici Histoire-Géographie en anglais). Des captures vidéo réalisées en salle multimédia ont permis d'observer certaines pratiques numériques des lycéens. Les vidéos obtenues ont ensuite été l'objet d'entretiens individuels semi-guidés avec 5 élèves volontaires.

\subsection{Atelier de production écrite}

Une séance en salle multimédia a été organisée avec 17 élèves de Première. Chaque élève avait pour consigne de rédiger un texte sur le voyage en Angleterre auquel ils avaient participé quelques semaines auparavant. Il leur avait été demandé de rédiger un brouillon en français avant la séance. Les élèves ont ainsi sélectionné leurs idées avant d'arriver à l'atelier et ont pu consacrer davantage de temps à la génération du texte en anglais [2]. Il a été demandé aux élèves d'ouvrir le traducteur en ligne de leur choix avant d'entamer la rédaction du texte et d'utiliser ce traducteur au moins une fois. Ils pouvaient avoir recours à tous les outils numériques qu'ils avaient l'habitude d'utiliser. Leur activité a été enregistrée par le biais du logiciel Flashback Express, qui permet les captations vidéo. En raison de quelques problèmes techniques, seules 15 vidéos ont été enregistrées. Les textes produits sur traitement de texte ont également été recueillis.

\subsection{Entretiens individuels}

A la suite de cette séance, 5 élèves volontaires ont participé à des entretiens individuels semi-guidés avec l'auteure de cet article, professeure d'anglais, mais qui n'était pas l'enseignante de la classe. Les élèves ont visionné la capture d'écran qu'ils avaient réalisée lors de l'atelier avec l'auteure. Ils avaient pour consigne d'expliquer et de commenter le plus librement possible leur utilisation des traducteurs en ligne et de façon plus générale leur recours aux ressources numériques dans le cadre d'une activité de production écrite. Lorsque la vidéo s'y prêtait, l'auteure a posé des questions leur demandant d'expliciter leur démarche.

Les entretiens ont été enregistrés par le biais du même logiciel utilisé lors de l'atelier, dont l'une des fonctionnalités est l'enregistrement du son à partir du micro de l'ordinateur. Grâce à ce procédé, les propos des participants ont pu être enregistrés en synchronisation avec la capture d'écran qu'ils commentaient. Les entretiens ont ensuite été retranscrits.

\section{Résultats et analyse}

Les transcriptions obtenues ont d'abord donné lieu à une analyse de contenu, puis la multimodalité des enregistrements des entretiens (la prosodie et le rythme de l'énonciation en particulier) a été prise en compte dans leur analyse. 


\subsection{Analyse de contenu}

Les transcriptions ont été analysées de plusieurs façons. Les mots à sémantisme plein ont tout d'abord été classés selon leur fréquence (cf. Tableau 1). Cette méthode ne permet pas en elle-même d'analyser les propos des participants mais constitue une première étape intéressante dans l'analyse d'un corpus, dans le sens où elle fait apparaître des singularités propres à chaque individu et oriente le regard du chercheur qui peut ensuite recontextualiser les mots en question.

Tableau 1. Classement des mots lexicaux employés par les élèves selon leur fréquence

$\begin{array}{ccccc}\text { Yacine } & \text { Djibril } & \text { Sarah } & \text { Anaïs } & \text { Nora } \\ \text { anglais } & \text { moi } & \text { crois } & \text { sais } & \text { mot(s) } \\ \text { mot(s) } & \text { phrase } & \text { phrase } & \text { mots } & \text { anglais } \\ \text { juste } & \text { texte } & \text { sais } & \text { anglais } & \text { français } \\ \text { directement } & \text { changé } & \text { savais } & \text { français } & \text { phrase } \\ \text { français } & \text { pense } & \text { mais } & \text { traducteur } & \text { sens }\end{array}$

\subsubsection{Djibril: " Pour moi, cette phrase, elle me paraît correcte. »}

Le mot le plus fréquemment employé par Djibril est «moi»(14 occurrences). La recontextualisation de ce mot fait apparaître des régularités intéressantes. En effet, sur les 14 occurrences du mot, on peut comptabiliser 8 occurrences de l'expression «pour moi ». Dans 2 autres cas, « moi » est employé dans l'expression « par rapport à moi ».

a. « Pour moi, la phrase que Reverso m'avait traduit, ben elle était pas correcte. »

b. «Parce que pour moi, 'fin..., j'l'ai toujours dit comme ça. On met après, le nom du stade et stadium. Après, je sais pas si c'est juste. Pour moi, c'était euh... comme ça qu'il fallait mettre. »

c. «Il me semble l'avoir entendu, mais j'suis pas sûr. Mais sinon pour moi, on pourrait dire aussi the stadium of Manchester United. Pour moi, les deux, c'est correct. »

d. «J'voulais dire "fais partie de mes équipes favorites", mais pour moi, l'expression qu'on m'a ressortie, en anglais, 'fin..., pour moi, c'était pas juste. »

e. « Pour moi, cette phrase, elle me paraît correcte. »

Dans chacun de ces énoncés, Djibril évalue la correction linguistique du texte produit par le traducteur: " correct » est répété dans 3 des 5 énoncés (a, c, e), «juste » est employé dans les deux autres énoncés (b,d). Mais cette évaluation est incertaine: « je sais pas si c'est juste » (énoncé b), «j'suis pas sûr» (énoncé c) nuancent les assertions « Pour moi, c'était euh... comme ça qu'il fallait mettre » (énoncé b) et «Pour moi, les deux, c'est correct» (énoncé c). En utilisant de façon répétée l'expression «pour moi », Djibril renforce sa présence énonciative. L'expression joue ici un rôle modalisant fort, parfois encore accentué par d'autres structures modalisantes: « il me semble » (énoncé c), " elle me parait » (énoncé e). Tous ces éléments laissent à penser que Djibril n'ose pas se montrer trop assertif lorsqu'il évalue la grammaticalité d'une phrase ou d'une expression en anglais ou la validité lexicale d'un mot: son discours n'est pas celui d'un expert. Cette surmodalisation des commentaires linguistiques pourrait être la trace d'un manque de confiance de l'élève envers ses compétences en anglais. L'attention portée à la correction morpho-syntaxique du texte produit laisse à penser que l'émotion ressentie par Djibril relève des émotions d'accomplissement, selon les termes de Pekrun et Linnenbrink-Garcia [14]: Djibril est préoccupé par le résultat de l'activité davantage que par l'apprentissage en lui-même. L'enjeu pour lui est avant tout d'écrire un texte «correct» et de ne pas faire d'erreurs. Cette émotion est peut-être d'autant plus forte que Djibril est en train de parler avec une enseignante d'anglais. Ce manque de confiance pourrait alors être interprété comme la 
crainte de dire des erreurs devant une experte et serait lié au contexte interactionnel de l'entretien.

\subsubsection{Sarah: "J'hésite."}

Parmi les mots les plus fréquemment employés par Sarah se trouve le verbe « savoir» conjugué au présent (14 occurrences) et au passé (14 occurrences). Contrairement à Anaïs, pour qui l'emploi de ce verbe relève essentiellement du tic de langage («je sais pas pourquoi », « je sais pas, en cours, c'est plus bizarre »), l'analyse de ce verbe en contexte révèle que Sarah l'utilise principalement dans son sens plein, soit parce qu'elle se remémore l'atelier d'écriture («Je sais plus ce que j'ai choisi»), soit parce qu'elle parle de ses connaissances. Elle peut alors employer le verbe «savoir» à la forme négative ou à la forme affirmative. Dans ce dernier cas, elle en atténue la portée à deux reprises par la conjonction de coordination « mais »:

a. «je savais que c'était every year, mais après des fois, je recherchais des mots parce que j'étais pas trop sûre si c'était forcément ça. »

b. « à chaque fois que j'écris, je sais pas si je choisis I like ou I love. J'hésite. »

c. « Et après, "qui habite là-bas", je savais pas comment le rajouter, après. »

d. « je savais que c'était one for years, du coup, je voulais voir un synonyme. »

e. «Quand je sais la phrase, je fais ma phrase en anglais, et après, je vais vérifier sur Reverso.»

f. «Je savais plus comment ... Je savais que le mur, c'était the wall, mais c'était "lamentations" que ... ça modifiait du coup. »

g. «Parce qu'en fait, les mots, je les sais, mais c'est plutôt pour les formulations de phrase. »

Le contexte nous permet ici de constater que l'expression « je sais pas » renvoie moins à l'ignorance de Sarah qu'à des doutes, des incertitudes exprimées également par les expressions «J'hésite » (énoncé b) ou «J'étais pas trop sûre » (énoncé a). Cette dernière expression est employée 6 fois au cours de l'entretien au présent ou au passé. Ces doutes ne portent pas sur le fait d'avoir écrit un texte juste ou faux, mais sur des points sémantiques (énoncés b et e) ou syntaxiques (énoncé c et g) et expliquent certainement pourquoi Sarah a besoin de "vérifier sur Reverso », même quand elle "[sait] la phrase » (énoncé e). L'incertitude de Sarah pourrait alors être considérée comme une émotion épistémique, centrée sur le savoir, dans le sens où elle est ici liée à une certaine conscience métalinguistique. L'énoncé $\mathrm{d}$ semble confirmer cette hypothèse, dans la mesure où Sarah explique que sa consultation de Reverso est motivée par la volonté d'employer une nouvelle expression.

L'analyse de contenu des transcriptions d'entretien donne ainsi accès à un certain nombre de traces des émotions ressenties par les apprenants, mais ces émotions sont complexes et bien que l'on puisse émettre des hypothèses sur leur objet, il est difficile d'identifier celui-ci avec certitude. Une analyse de données multimodales peut permettre d'enrichir l'analyse et d'entrevoir les émotions à l'œuvre dans le processus d'écriture et dans le recours aux traducteurs en ligne. C'est le cas pour Anaïs et Sarah.

\subsection{Analyse de données multimodales}

La mise en relation des commentaires de l'élève participant à l'entretien et de la capture d'écran réalisée lors de l'atelier d'écriture peut offrir un éclairage intéressant sur les enjeux affectifs relatifs aux usages des traducteurs en ligne et à l'apprentissage de l'anglais en milieu institutionnel. Nous allons nous pencher sur deux extraits d'entretiens qui montrent 
de quelle façon l'observation de pratiques de la part de deux élèves peut les aider à mettre leurs émotions à distance ou au contraire en générer de nouvelles.

\subsubsection{Anaïs: "En anglais, j'y arrive pas trop, trop."}

Lors de l'entretien, une question posée par l'auteure à propos de ce qui se passe à l'écran entraîne un commentaire plus général portant à la fois sur les compétences linguistiques d'Anaïs et sur sa connaissance des traducteurs en ligne (cf. Tableau 2).

Tableau 2. Transcription d'un extrait de l'entretien avec Anaïs

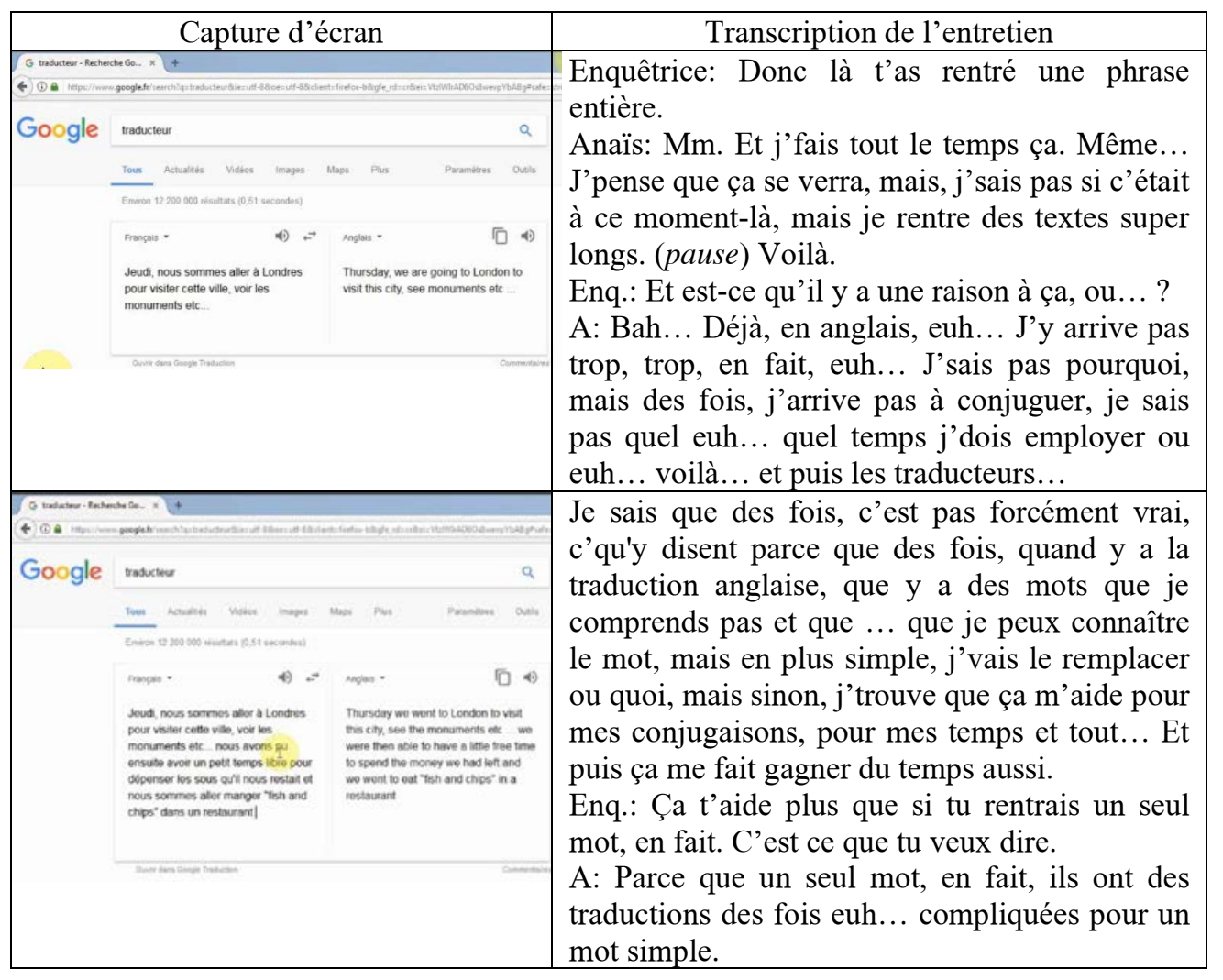

On peut constater qu'Anaïs répond tout d'abord à une remarque de la part de l'enquêtrice à propos de ce qu'elles observent toutes les deux à l'écran (« Donc là, tu rentres une phrase entière. »). Anaïs confirme qu'il s'agit d'une pratique habituelle, puis le propos glisse immédiatement de ce qui est en train de se passer à l'écran vers un discours plus général sur les pratiques d'Anaïs.

Ce discours est assez nettement scindé en deux parties: la première porte sur les compétences d'Anaïs en anglais ou plus précisément sur la perception qu'elle en a, tandis que la deuxième partie est centrée sur son usage de Google Traduction. Lorsqu'Anaïs évoque ses difficultés en anglais, elle est capable d'identifier les difficultés qu'elle rencontre. Celles-ci sont clairement de nature grammaticale (conjugaison des verbes, choix des temps). Le débit est lent, les hésitations sont présentes, mais le ton reste neutre: la charge émotionnelle semble modérée. Il est difficile de dire si les difficultés rencontrées sont source de gêne, d'angoisse ou de frustration. Il est possible qu'elles ne provoquent pas d'émotions particulières. Toutefois, étant donné qu'Anaïs a choisi de s'inscrire en section 
européenne, il est peu probable que le fait qu'elle n'exprime pas d'émotions de façon explicite puisse être interprétée comme un désintérêt pour la discipline. Il est possible ici que l'écran aide Anaïs à mettre à distance les émotions qu'elle ressent en lien avec l'apprentissage de l'anglais, comme si l'écran rendait possible un dédoublement entre le sujet observé (Anaïs écrivant un texte) et le sujet observant (Anaïs commentant la vidéo réalisée en salle multimédia).

Le changement de ton est très net lorsqu'Anaïs explique le fonctionnement des traducteurs en ligne: le débit s'accélère, les hésitations disparaissent. La dernière phrase est assertive, les marqueurs de modalisation sont quasi inexistants. La combinaison de ces différents marqueurs révèle une forme d'assurance et laisse à penser qu'Anaïs considère avoir développé une certaine expertise empirique des traducteurs en ligne. Le développement de compétences numériques pourrait alors compenser les fragilités linguistiques ressenties par Anaïs.

\subsubsection{Sarah: "ça me stresse, ce que je fais, là."}

Lorsque Sarah observe sa production (cf. Figure 2), elle réagit ainsi:

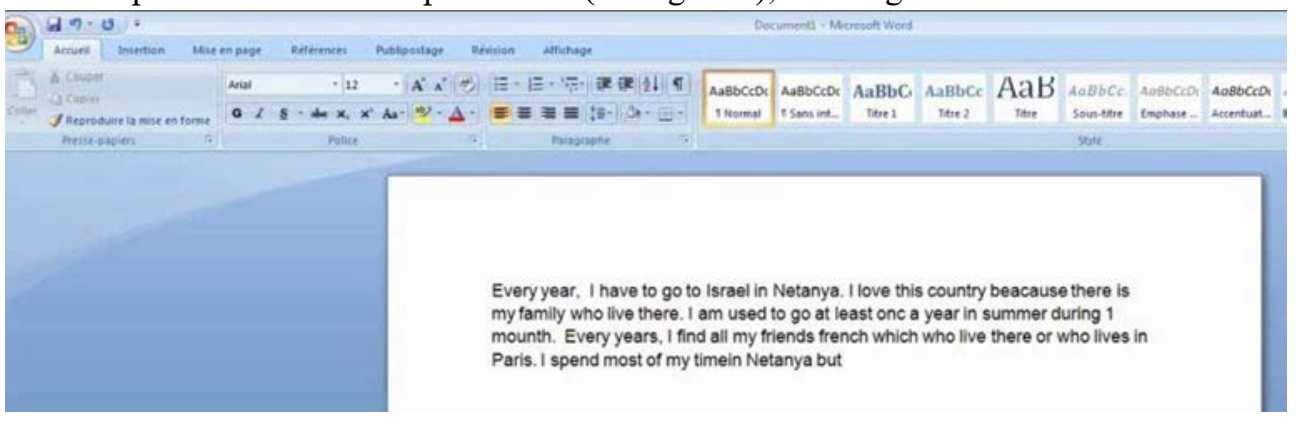

Fig. 2. Capture d'écran commentée par Sarah

Sarah: Mais... ça me stresse, c'que j'fais, là. (sourire)

Enq.: (rire)

Silence.

Enq.: Après, qu'il y ait des fautes de frappes, euh... ça, c'est normal. On voit bien que le timein, là, c'est une faute de frappe.

S: Même le which / who, là aussi, là... c'est horrible.

Sarah éprouve un vif embarras lorsqu'elle découvre a posteriori les erreurs qu'elle a effectuées. Bien que l'enregistrement laisse entendre son sourire, le sémantisme de ces commentaires est sans ambigüité: l'exercice est source de stress et l'erreur qu'elle identifie est « horrible». Alors que la capture d'écran semblait aider Anaïs à mettre à distance certaines émotions, l'observation de l'activité joue pour Sarah le rôle d'un stimulus et provoque une nouvelle émotion négative (inconfort, gêne ou honte). Il est toutefois difficile de déterminer si c'est l'erreur en elle-même qui est à la source de cette émotion négative, le fait que ce soit elle qui ait fait l'erreur et qu'il est trop tard pour la corriger, ou bien le fait qu'elle n'est pas seule à regarder cette vidéo et à voir cette erreur.

En réponse à cette émotion, l'enquêtrice rit et s'efforce de dédramatiser l'erreur en insistant sur le fait que les fautes de frappe sont un phénomène courant (« c'est normal ») et que ces fautes sont distinctes des erreurs linguistiques. Les éléments para-verbaux (rire) et non-verbaux (sourire) semblent révéler à ce moment de l'entretien une connivence entre Sarah et l'enquêtrice et la gestion de l'émotion se fait ici au sein de l'interaction.

\section{Discussion}


La réalisation de captures d'écran vidéo répondait à un objectif double: il s'agissait d'une part d'observer les pratiques réelles des lycéens et d'obtenir un objet de discussion permettant aux élèves de verbaliser leurs émotions dans le cadre d'entretiens. Les vidéos obtenues ont effectivement permis aux élèves d'objectiver et de verbaliser leurs émotions, mais elles ont aussi provoqué l'émergence de nouvelles émotions chez l'élève, très certainement en lien avec la forme de l'entretien.

\subsection{L'observation de l'activité comme mise à distance des émotions}

A travers l'observation d'une activité singulière, c'est bien souvent sur leurs pratiques et sur la relation qu'ils entretiennent avec l'anglais que les participants ont axé leurs discours. Ils ont exprimé des émotions diverses, mais il semble que les émotions les plus fréquentes soient des émotions négatives qui renvoient au doute et au manque de confiance en leurs compétences en anglais et à la crainte de l'erreur. Ces affects sont verbalisés, mais la charge émotionnelle apparaît peu dans les discours: l'écran semble aider les participants à se dédoubler entre un sujet observé, qui écrit et utilise les traducteurs en ligne, et un sujet observant, qui n'est pas engagé personnellement dans l'observation. Cette dissociation parfois troublante permet à des participants comme Anaïs de mettre certains affects à distance, de les objectiver, et fait écho à la relation intersubjective entre sujet et objet de la recherche, fondamentale à tout processus de connaissance selon Piaget [17].

Doute, anxiété, manque de confiance, frustration: force est de constater qu'il est souvent difficile d'identifier précisément l'émotion ressentie par l'élève ainsi que son objet. La tâche est d'autant plus difficile que plusieurs émotions peuvent être ressenties en même temps et que l'on n'a pas toujours conscience des émotions qui nous traversent [8]. On peut toutefois considérer que la classification des émotions n'est pas une finalité en soi, l'enjeu étant ici de comprendre le rôle joué par les émotions dans l'utilisation des traducteurs en ligne. L'observation des vidéos par les participants leur permet de prendre conscience de certaines d'entre elles, mais elle en suscite également de nouvelles.

\subsection{L'émergence de nouvelles émotions chez le sujet observant}

Si la vidéo a permis une objectivation des émotions du sujet observé pour certains participants, elle a également provoqué des émotions nouvelles chez d'autres en tant que sujet observant. Les variations de ton sont assez nettes entre l'expression des premières et des secondes: le ton reste souvent assez neutre dans le premier cas tandis que dans le second, le débit, la prosodie ou bien des marqueurs para-verbaux peuvent indiquer les émotions du sujet observant. Il peut s'agir d'émotions positives, telles l'assurance des participants lorsqu'ils évoquent leur expertise empirique des traducteurs en ligne, ou bien des émotions négatives, comme l'embarras de Sarah qui évalue sa production écrite.

Les émotions négatives ne portent toutefois pas nécessairement préjudice à l'apprentissage. Selon Pekrun et Perry [15], les émotions positives sont certes plus propices aux prises de risque et aux opérations de haut niveau, facilitant ainsi un apprentissage plus profond, mais les émotions négatives sous-tendent les opérations de bas niveau et les activités plus analytiques. En outre, les émotions ressenties ici par Sarah sont peut-être d'autant plus fortes qu'elle n'est pas seule à observer son activité. Il est possible que la présence d'une enseignante d'anglais qui voit ses erreurs en même temps qu'elle intensifie le sentiment d'embarras ou de honte dont elle fait l'expérience.

\subsection{L'entretien comme interaction: des émotions situées}


Qu'il s'agisse de Sarah ou de Djibril, il est probable que la conscience d'un regard extérieur influence les émotions qu'ils ressentent. L'anxiété de Djibril, qui hésite à se positionner sur la validité linguistique des phrases produites par le traducteur en ligne, de même que la gêne de Sarah devant certaines erreurs ne sont peut-être pas suscitées seulement par l'observation de la vidéo. Il est possible qu'elles soient provoquées ou intensifiées par l'environnement des participants, c'est-à-dire ici par la présence d'une interlocutrice experte. Les émotions ressenties ne portent pas sur la relation avec un tiers et ne sauraient donc être décrites (seulement) en termes d'émotions «sociales», pour reprendre la classification de Pekrun \& Linnenbrink-Garcia [14], mais ces éléments d'analyse prouvent que les émotions doivent être considérées comme des expériences intersubjectives, influencées par les interactants [9]. La méthodologie adoptée, c'est-à-dire la conduite d'entretiens entre un élève et un expert, constitue alors un biais qu'il est nécessaire de prendre en compte lorsque l'objectif est d'étudier les mécanismes émotionnels à l'œuvre chez l'apprenant dans le cadre de l'apprentissage de l'anglais. L'observation d'interactions entre pairs dans le cadre d'ateliers d'écriture en binômes pourrait constituer une alternative intéressante pour étudier les traces des mécanismes émotionnels à l'œuvre lorsque les lycéens ont recours aux traducteurs en ligne. Les enjeux affectifs inhérents à toute interaction seraient toujours présents mais l'influence de l'asymétrie entre experts et apprenants serait réduite.

\section{Conclusion}

Bien que l'analyse des émotions reste ouverte à de nombreuses interprétations, une analyse des entretiens qui s'appuie sur les dimensions verbale, prosodique et rythmique ainsi que sur la vidéo aide à mieux cerner la complexité des affects qui entrent en jeu lorsque les lycéens ont recours aux traducteurs en ligne. Le besoin de vérification peut être relié à un manque de confiance de la part de certains lycéens envers leurs compétences linguistiques (Sarah) ainsi qu'à une appréhension de l'erreur (Djibril). On pourra toutefois objecter que les vérifications effectuées par les participants ne sont pas toujours liées à des émotions négatives et que la crainte de se tromper peut se combiner à la curiosité et au désir d'accroître ses connaissances. Le traducteur jouerait alors à la fois un rôle d'aide cognitive et de soutien affectif, d'autant plus que l'assurance que les lycéens ressentent envers certaines de leurs compétences numériques pourrait contrebalancer leur insécurité linguistique, comme semble l'indiquer l'exemple d'Anaïs [18].

L'affect joue un rôle indéniable dans l'apprentissage et même si les émotions négatives peuvent inciter l'apprenant à faire preuve d'une plus grande attention et de davantage de précision, ce sont plutôt les émotions positives, telles le plaisir et la curiosité, qui sont associées à une démarche créative et à des stratégies de compréhension plus profondes et plus propices à l'apprentissage. On ne peut toutefois nier que l'apprentissage implique souvent des émotions négatives ou tout du moins un inconfort, dans la mesure où celui-ci s'appuie nécessairement sur un déséquilibre entre la nouveauté et les savoirs antérieurs [19]. Ce déséquilibre est d'autant plus fort lorsqu'il s'agit de l'apprentissage d'une langue étrangère, pour lequel l'apprenant doit recréer ses ancrages symboliques et affectifs [20]. Il revient alors à l'enseignant "d'inscrire ce déséquilibre dans un contexte de sécurité favorable à la régulation des émotions générées par l'activité d'apprentissage. » [19].

Les traducteurs en ligne sont des outils rassurants mais complexes: ils incluent désormais souvent des dictionnaires unilingues et bilingues, des dictionnaires de synonymes ainsi que des exemples contextualisés et requièrent une expertise linguistique en L1 et en L2 dont ne disposent pas toujours les lycéens. Leur adoption massive par les lycéens nous invite aujourd'hui à mener une réflexion sur le rapport des apprenants à 
l'erreur et sur une intégration raisonnée des traducteurs en ligne dans l'apprentissage de l'anglais en milieu institutionnel afin d'aider les élèves tant sur un plan cognitif qu'affectif.

\section{Bibliographie}

1. C. Del Olmo Comprendre les enjeux des stratégies d'apprentissage pour devenir enseignant de FLE. Recherche et pratiques pédagogiques en langues de spécialité Cahiers de l'Apliut. 35, 1 (2016)

2. L. Flower, J. Hayes A Cognitive Process Theory of Writing. College Composition and Communication. 32, 4, 365-87 (1981)

3. W. James The principles of psychology. (Mcmillan, London, 1890)

4. N. Frijda The laws of emotion. American psychologist 43, 5, 349-58 (1988)

5. E. Linnenbrink Emotion research in education: theoretical and methodological perspectives on the integration of affect, motivation, and cognition. Educational Psychology Review 18, 4, 307-314 (2006)

6. C. Plantin Micro-émotions en interaction: «ah merde, ya rien pour maman». Voix Plurielles. 12, 1, 5-21 (2015)

7. A. Damasio L'erreur de Descartes: la raison des émotions (Éditions Odile Jacob, Paris, 1995)

8. A. Tcherkassof, N. Frijda Les émotions: une conception relationnelle. L'Année Psychologique 114, 3, 501-535 (2014)

9. C. Kerbrat-Orecchioni Quelle place pour les émotions dans la linguistique du XXe siècle? Remarques et aperçus. In: Les émotions dans les interactions, $50-51$ (Presses universitaires de Lyon, Lyon, 2000)

10. S. Benesch Considering Emotions in Critical English Language Teaching: Theories and Praxis (Routledge, New York, 2012)

11. R. Pekrun, T. Goetz, A. Frenzel, P. Barchfeld, R. Perry. Measuring emotions in students' learning and performance: The Achievement Emotions Questionnaire (AEQ). Contemporary Educational Psychology 36, 1, 36-48 (2011)

12. K. Orlova, J. Ebiner, P. Genoud Émotions et apprentissages scolaires: quand les représentations des enseignants permettent de mieux envisager des pistes de formation. Recherche formation 79, 2, p.28 (2015)

13. S. Crossley Technological disruption in foreign language teaching: The rise of simultaneous machine translation. Language Teaching 51, 4, 541-552 (2018)

14. R. Pekrun, L. Linnenbrink-Garcia. International handbook of emotions in education. (Routledge, New York, 2014)

15. R. Pekrun, R. Perry Control-value theory of achievement emotions. In: International handbook of emotions in education. (Routledge, New York, 2014)

16. G. Trevors, K. Muis, R. Pekrun, G. Sinatra, M. Muijselaar. Exploring the relations between epistemic beliefs, emotions, and learning from texts. Contemporary Educational Psychology 48, 116-132 (2017)

17. M. Uhl Le sujet oublié de la sociologie. Connexions, 89, 96 (2008)

18. M-C. Penloup Littératies numériques : quels enjeux pour la didactique de l'écriturelecture ? Réponse à Jeannine Gerbault. Recherches en didactique des langues et des cultures Les cahiers de l'Acedle 9, 9-2 (2012) 
19. F. Cuisinier Émotions et apprentissages scolaires: quelles pistes pour la formation des enseignants ? Recherche \& formation. 81, 1, 9-21, p.13 (2016)

20. E. Piccardo Évolution épistémologique de la didactique des langues : la face cachée des émotions. Lidil Revue de linguistique et de didactique des langues 48, 17-36 (2013) 